\title{
The burden of colorectal cancer: prevention, treatment and quality of services
}

\author{
Panos Kanavos • Willemien Schurer
}

Published online: 10 December 2009

(C) Springer-Verlag 2009

JEL Classification $\mathrm{I} 11 \cdot \mathrm{I} 12 \cdot \mathrm{I} 18$

\section{Background}

Colorectal cancer (CRC) is one of the most common forms of cancer and is a substantial health burden in most developed countries, with high incidence and mortality in combination with moderate survival. It is often diagnosed late due to the absence of screening programmes, lack of knowledge about symptoms and/or a reluctance to seek medical help once the symptoms have appeared. Although improvements in outcomes over time have been made, compared with other high incidence cancers, such as breast cancer, overall survival for CRC remains relatively poor. However, early detection is feasible and for patients with early disease, surgery alone can be curative. Chemotherapy is essential to provide a chance of cure for patients with more advanced disease and during the past 10 years, a number of new agents have been introduced, with a subsequent increase in life expectancy for patients with StageIV disease from 5 to over 20 months. However, facilities for treatment, use of and adherence to clinical management guidelines and access to new, effective treatments reportedly vary widely across OECD countries. Cancer spending on CRC is difficult to disaggregate, but evidence suggests that treatment costs are high, particularly for Stage III or IV which are advanced stages of the disease. Most countries

P. Kanavos $(\bowtie) \cdot$ W. Schurer Department of Social Policy and LSE Health, London School of Economics, Houghton Street, London WC2A 2AE, UK

e-mail: p.g.kanavos@1se.ac.uk also appear to have, for the most part, little concerted effort with regards to screening activities. Similarly, both public and political interest in CRC activities appears minimal to non-existent in some countries compared to the public/ media profile of breast cancer, for example.

\section{The rationale for this supplement}

Although CRC burden varies within countries, in Europe it is the second most diagnosed form of cancer, accounting for $13 \%$ of total cancer incidence, and it is also second in cancer mortality, accounting for $12 \%$ of total cancers in 2006 [1]. Over the past decade, incidence in developed countries has been increasing, and is most likely to continue to do so with ageing populations, while mortality has decreased somewhat but not as much as in other similar high-incidence, high-mortality cancers.

Incidence and mortality differ significantly between European countries, even countries that are geographically close to each other. Southern Europe has the lowest incidence and mortality (Greece, Turkey), while Eastern Europe has the highest (Czech Republic, Hungary, Slovakia), probably due to differences in lifestyle, stage at diagnosis and treatment. Similarly, males have higher incidence and mortality than females, relating to differences in screening participation, physician-seeking behaviour as well as lifestyle. These variations suggest that country policy and societal norms play a large role in CRC development and ultimately cancer burden.

Although survival from CRC has increased over the past decade, largely due to improved treatments and standards, it still remains poorer in Europe than in North America. Evidence indicates that less than 55\% of Europeans achieve 5-year CRC survival in comparison to over $75 \%$ 
for European breast and prostate cancer patients and 65\% for American CRC patients [2, 3]. These figures vary between countries from under $40 \%$ (Poland) to almost $60 \%$ (Sweden), with women having a slightly better survival than men. Part of the problem is the high detection of later stages of cancer versus early detection found in other cancers with established screening programmes, poor or irregular surveillance practices of those at increased risk, as well as limited resources allocated to CRC.

The above trends have acted as a stimulus to policymakers at the European level, particularly with regards to taking some action on prevention. In 2003, the EU Council recommended including national CRC screening [4], whereas in 2007 the Brussels Declaration re-iterated the need for greater official support for screening and standardisation of practices across Europe [5]. Some EU member states are also more active than others in adopting policies and strategies to manage the burden of CRC. However, in the majority of countries the interest in CRC and its management, both political and public, seems to be limited.

\section{Issues for research}

The papers in this supplement debate a range of issues relevant to $\mathrm{CRC}$ management and its treatment in a number of OECD, mostly European, countries plus the Russian Federation. The first issue is the level of resources allocated to cancer in general and to CRC in particular; this would provide a signal on the priorities individual countries have in place with regards to management of the disease from prevention to diagnosis to treatment. Yet, information on CRC resource allocation is particularly scarce.

The second issue is to discuss the uptake of screening and surveillance, with a view to understanding the policies, practices and priorities of different countries, to what degree are preventative policies implemented, how committed governments are to these priorities, what are the driving forces behind these and how strong patient participation is. It is known that CRC is one of the few cancers amenable to cancer-screening activities and if detected at its precancerous adenomatous polyp stage it can be prevented. As this cancer is also largely asymptomatic until later stages, screening can detect early-stage cases with much higher rates of curative treatment and longer survival. Yet, available evidence suggests that despite these facts and in addition to rising CRC incidence, only a minority of countries participate in formal CRC screening.

The third issue relates to the identification of the actual resource and capacity implications for countries to be able to perform screening as well as surveillance. Some of the issues involved here concern budgets, but also human resource management, particularly the availability of endoscopy centres and endoscopists and whether nurse endoscopists are used to perform endoscopies.

The fourth issue is the availability of and access to medical treatment as well as the uptake of new techniques and access to them. The elements explored in this context are, among others, whether there are geographical disparities in access to treatment, particularly for rural or remote areas, whether CRC treatment is available and accessible to all socioeconomic groups, whether standards exist in individual countries and how they can be compared, whether there are practice variations and why, what the relevant waiting times are - if any - from diagnosis to treatment and follow up and what the relevant out-ofpocket payment arrangements are for patients.

The fifth issue is availability of and access to pharmaceutical treatment and also relates to the uptake of new medical technologies. Clearly, there have been a number of advances in oncology pharmaceutical treatments from oral versions of traditional 5-fluorouracil therapy to additional cytotoxic chemotherapy and new targeted biological treatments. This means more choice is available for patients and oncologists. Many of these reduce the significant risk of recurrence found with CRC, while others significantly increase the survival rate. The papers in this supplement explore whether there are variations in pharmaceutical therapy within as well as across countries and why, and discuss the rate of uptake of new treatments.

\section{Objectives}

The supplement aims to capture the current issues and debate surrounding the management and funding of CRC in a number of OECD countries and the Russian Federation. This allows an accurate picture of the current state of colorectal cancer management to be formulated and trends in practice to be identified.

By exploring the issues outlined in the previous section, the overall objectives of the supplement are, (1) to understand the available data sources for CRC at national level, their accessibility, availability, and use in research and policy; (2) to map the funding and resource allocation processes on cancer in general and CRC in particular; (3) to identify the extent of primary and secondary (screening) prevention policies for $\mathrm{CRC}$, and the extent to which these are actively encouraged, implemented and enforced in the study countries; (4) to review the differences in cancer survival between countries and to discuss the effectiveness of health-care provision from a health-system perspective; (5) to discuss the available standards of care for CRC at national and international level; (6) to determine the ability and willingness of hospitals to implement national 
guidance and to examine current pathways for CRC management; (7) to assess the access to and the availability of diagnostic services and pharmaceutical treatments for CRC. And, finally, (8) to investigate the appropriateness and adequacy of current funding and management of CRC services.

In order to achieve the above objectives and to ensure homogeneity in the papers presented in this supplement, a template was developed requesting data and information on each of the above-mentioned themes. This was validated externally with the assistance of some of the authors and was subsequently administered to all authors. The papers presented in the supplement reflect national policies and strategies in the management of CRC.

Acknowledgments We are grateful to all the authors that have contributed to this effort to provide an accurate account of the state of the art in the management of CRC in the countries included in this supplement. We have gained significantly from this interaction, which has also enabled us to understand the wider health-policy implications facing each country. We are also grateful to the editor-in-chief of this journal and his willingness to provide feedback and solutions at all stages in the preparation of this supplement. Finally, we are indebted to F. Hoffmann La Roche Ltd for supporting the publication of this supplement by an unrestricted educational grant.

Conflict of interest statement The authors do not report any conflict of interest associated with this paper.

\section{References}

1. Ferlay J, Autier P, Boniol M, et al.: Estimates of the cancer incidence and mortality in Europe in 2006. Ann. Oncol. 18(3), 581-592 (2007)

2. Verdecchia A, Francisci S, Brenner H, et al.: Recent cancer survival in Europe: a 2000-02 period analysis of EUROCARE-4 data. Lancet Oncol. 8(9), 784-796 (2007)

3. Sant, M., Aereleid, T., Berrino, F., et al.: EUROCARE-3: survival of cancer patients diagnosed 1990-94-results and commentary. Ann. Oncol. 14(S5), v61-v118 (2003)

4. Council of the European Union: Council recommendation of 2 December 2003 on cancer screening. Official J. Eur. 327, 34-38 (2003)

5. Europe against Colorectal Cancer: declaration of Brussels. www.future-health-2007.com (2007). Accessed June 2009 